\title{
ESTIMATION OF THE MEAN ENERGY OF MUONS IN MULTILAYER DETECTORS
}

\author{
T.T.Barnaveli, Yu.G.Werbetsky, I.V.Khaldeeva, N.A.Eristavi. \\ Institute of Physics, Georgian Acad. Sci., \\ Tamarashvili str. 6, Tbilisi - 380077, Republic of Georgia. \\ (E-mail: bart@physics.iberiapac.ge).
}

\begin{abstract}
The technique of muon mean energy determination in multilayer detectors is developed. The mean energy is measured by means of average small bursts $m$ i.e. the number of electrons and positrons generated by muons in the detecting layers of device via three basic processes - creation of $e^{+} e^{-}$pairs, $\delta$-electrons and bremsestrahlung. The accuracy of the method is considered.
\end{abstract}

Key words: muon energy, multilayer detectors.

\section{Introduction.}

For investigation of the penetrating component of cosmic radiation by means of multilayer detectors it is essential usually to estimate the energy of registered events. This task in cosmic ray physics faces the serious technical obstacles, especially for large detectors and for muons of very high energies. However many problems in cosmic ray physics can be solved if one restricts to the estimation of the mean energy of muons in samples of events of different types, e.g. in muon groups of some fixed multiplicity $n$. The method of muon mean energy estimation is evaluated below.

This method is based on the analysis of a small bursts accompanying the passage of penetrating particles through the layers of filter and detecting elements of device. These bursts are generated by muons in the filter layers of device via three basic processes - creation of $e^{+} e^{-}$pairs, $\delta$-electron emission and bremsestrahlung. The value of mean burst $m$ turned out to be very effective parameter to evaluate the mean energy of penetrating particles in multilayer detectors.

The high efficiency of penetrating particle registration and energy estimation is due to the circumstances quoted below. Despite the fact that the 
muon energy losses caused by the pair production process are close to those caused by the bremsestrahlung, the cross-section of the first-mentioned process is essentially higher. It increases with the muon energy value and at $E \geq 10^{12} \mathrm{eV}$ the probability of $e^{+} e^{-}$pair creation in the absorber layer of the optimal thickness is close to the unit. The thickness of the absorber layers is to be chosen with the account of the requirement of the minimization of particle number fluctuations in avalanches. The calculations [[] show that at the muon energies of the order of $10^{12} \div 10^{13} \mathrm{eV}$ the minimal dispersion is ensured with the thickness of lead absorber of $10 \mathrm{t}$-units, i.e. $\sim 5 \mathrm{~cm}$.

The approach stated below becomes most effective for the investigation of high energy muons by means of visual track method of observation. Visual observation allows to register even the smallest (single particle) bursts, to distinguish easily the events by their nature and to handle the rare events of high multiplicity. The influence of transition effects are simple to be taken into account since it is easy to separate the particles created in absorber from those created in the lid and the walls of spark chambers. The application of the multilayer system of spark chambers to the evaluation of muon energy was proposed earlier in [2].

Up to day in our experiments (e.g. [3]) we used the version of this approach described in [四]. In the present work some further development and verification of the method is proposed.

For the analysis of the approach the really working [3] multilayer spark detector is considered below. The detector consists of 8 layers of spark chambers separated by a $5 \mathrm{~cm}$ thick lead absorbers. The area of each layer of the detector is $4.8 \mathrm{~m}^{2}$. The spark chambers are made of glass and contain neon of high purity at atmospheric pressure. The electrodes are made of $1 \mathrm{~mm}$ duraluminium. The detector is located under the rock at the depth of $190 \mathrm{hg} \cdot \mathrm{cm}^{-2}$. This determines the threshold energy of registered muons - about $35 \mathrm{GeV}$. The method proposed is in principle applicable for the systems containing the registering elements of any type (e.g. Heiger-Müller counters, neon tubes etc.)

In chapter 2 the main parameters used are considered. In chapters 3 and 4 the principle of muon mean energy estimation in multilayer detectors is justificated. Chapter 5 is dedicated to the questions of device calibration. In chapter 6 actually the process of muon energy estimation is described. The dependence $E(m)$ is given for the detector under consideration. 


\section{The main input parameters.}

In the real observations of high and superhigh energy cosmic ray muons the following parameters are usually available or easily measurable:

1. The mean threshold energy $T=T(x)$ of registered muons. This value depends on the thickness $x$ of the filter located over detector (rock, water etc.) and its absorbing features.

2. The exponent $\beta$ of the spectrum of accompanying bursts $Y(m) \propto$ $m^{-(\beta+1)}$. According to [5], in the case of power form of muon energy spectrum $P(E) \propto E^{-(\gamma+1)}$ and with the condition of equilibrium of accompanying electromagnetic cascade ( i.e. in the vicinity of avalanche maximum) the exponents $\beta$ and $\gamma$ approximately coincide.

3. The value of mean burst $m$ (i.e. the mean number of $e^{+}$and $e^{-}$accompanying the passage of penetrating particle through the multilayer system per one layer).

Note that the value of $m$ in the most of cases is determinable with much less error than $T$ and $\beta$.

If the differential energetic spectrum of penetrating particles registered under the filter is defined in normalized power form

$$
P(E \mid x)=\frac{\gamma}{T(x)} \cdot\left(1+\frac{E}{T(x)}\right)^{-(\gamma+1)},
$$

then their mean energy (under the filter)

$$
<E>=\int_{0}^{\infty} E \cdot P(E) d E
$$

is equal to

$$
<E>=\frac{T(x)}{\gamma-1}, \quad \gamma>1
$$

Now, if in (2.3) one accepts for valuations $\hat{\gamma}=\hat{\beta}$ (the conditions of such substitution correctness were given above in item 2), it becomes possible to determine the mean energy valuation $\hat{E}$ through the energetic threshold $\hat{T}$ and the exponent $\hat{\beta}$ of the differential spectrum of small bursts. However actually the conditions necessary for such application of (2.3) are not fulfilled. Even for the single muons the spectrum differs from (2.1); it is significantly 
flatter at low energies. On the other hand, for some samples of events it turns out that $\beta<1$. In this case the mean energy $\langle E\rangle$ formally is not defined at all. However $m$ in these samples is steadily recurring in the consecutive experiments, so in any case one can consider the value of mean burst $m$ as the well defined one. This means that the burst spectrum is also not of pure power-type. It gets steeper at the big bursts.

\section{Determination of mean energy of muons.}

The parametrization of muon energy $\left(E_{0}\right)$ spectra in atmosphere (both for single muons and for those which are a component of the events of any other type) will be taken in the normalized form [6]:

$$
P\left(E_{0}\right)=\frac{\gamma}{T_{0}} \cdot \frac{\left(1+E_{0} / T_{0}\right)^{-\gamma} \cdot\left(1+E_{0} / K_{0}\right)^{-1}}{{ }_{2} F_{1}\left(1,1 ; \gamma+1 ; 1-T_{0} / K_{0}\right)}
$$

Here ${ }_{2} F_{1}$ is the Gaussian hypergeometric function, $T_{0}$ - the characteristic energy describing flattening of the spectrum at low energies (of the order of $1 \mathrm{GeV}) ; K_{0}$ - the critical energy characterizing the competition of two processes - decay of pions and their interaction with air nuclei at the height of effective generation of muons []]. Generally speaking, the analogous process for $K$-mesons is to be taken into account as well. For this reason in (3.1) it is taken $K_{0} \simeq 100 \mathrm{GeV}$ - the effective critical energy approximately describing the evolution of the flux of $\pi$ - and $K$-meson mixture [6]. For a not very large values of filter thickness $x$ it is possible not to take into account the large fluctuations of muon energy losses and thus the mean energy losses may be taken in the standard form

$$
-\frac{d E}{d x}=a+b E
$$

where $a$ and $b$ are the parameters characterizing the material of the filter. In this case the form of the spectrum is conserved through the evolution of spectrum down the filter depth [8], while the initial energy $E_{0}$ of muons (the energy at the entry to the filter) is the integral of evolution. It is connected with the muon energy $E$ under the filter by the relation

$$
E_{0}=(C+E) e^{b x}-C,
$$

where $C=a / b$ is the parameter (energy dimensioned) characterizing the material of filter (for the small depths $x$ and small energies $E$ one obtains the usually used approximate expression $\left.E_{0} \simeq E+a x\right)$. 
The relation (3.3) describing the full evolution effect of the spectrum with respect to the depth $x$ makes it possible to re-write the spectrum of muons (3.1) under the filter in the form dependent on $E$ and analogous to (3.1):

$$
P(E \mid x)=\frac{\gamma}{T(x)} \cdot \frac{(1+E / T(x))^{-\gamma} \cdot(1+E / K(x))^{-1}}{{ }_{2} F_{1}(1,1 ; \gamma+1 ; 1-T(x) / K(x))} .
$$

Here the functions $T(x)$ and $K(x)$ describe the evolution of the characteristic energy $T_{0}$ and critical energy $K_{0}$ with the depth $x$ of the filter:

$$
\left.\begin{array}{r}
T \equiv T(x)=C-\left(C-T_{0}\right) e^{-b x} \simeq T_{0}+\left(a-T_{0} b\right) x ; \\
K \equiv K(x)=C-\left(C-K_{0}\right) e^{-b x} \simeq K_{0}+\left(a-K_{0} b\right) x .
\end{array}\right\}
$$

Further we will accept that the energy spectra in the samples of different kinds of events (in particular, differing by the number of muons in muon groups) may be described by means of functions of (3.4)-class. Here we take the natural assumption that energetic parameters $T$ and $K$ specified by the process of absorption in filter and by the $\pi$ - and $K$-decays at high altitudes are the same for all types of samples of events. We assume that the difference of spectra of these samples is described completely by the difference of values of their exponents $\gamma$. Now, once evaluating the value of $\gamma$ by means of any method one can estimate the mean energy of muon by the distribution (3.4):

$$
<E(x)>=\frac{T(x)}{\gamma-1} \cdot \frac{{ }_{2} F_{1}(2,1 ; \gamma+1 ; Z)}{{ }_{2} F_{1}(1,1 ; \gamma+1 ; Z)},
$$

where

$$
Z \equiv Z(x)=1-T(x) / K(x)
$$

\section{Evaluation of the effective exponent of the spec- trum.}

To evaluate the effective value of $\gamma$ we use the value of mean burst $m$ which is easy to obtain for the given sample of events. On the basis of [प, 9] the mean electromagnetic cascade accompanying the passage of muon of fixed energy $E$ through the layer of the absorber of the optimal thickness was numerically calculated. The obtained $E$-dependence of $\bar{m}$ can be approximated as

$$
\bar{m}(E)=B \cdot(E / \epsilon)^{\alpha},
$$


where $\bar{m}$ is an average burst at fixed energy $E$ of the muon; $\epsilon \equiv 1 G e V$ - is the energy scale; the parameters $\alpha, B$ are determined by the properties and the size of the absorber in the detector. The exponent $\alpha$ one can estimate through the numerical calculations with sufficiently high accuracy. The value of the normalization parameter $B$ one has to estimate through calibration of the device by the well known flux of a single cosmic ray muons (see further). Of course, the more accurate results can be achieved by measuring the relation like (4.1) with a monochromatic beam of muons from accelerator at a different energies. However, only the first way is practically available in cosmic ray experiments.

Averaging the approximate relation (4.1) for the $\bar{m}$ value over the spectrum $P(E \mid x)$ of muon energies ([3.4) one obtains for the twice-averaged burst to be used further again:

$$
\begin{array}{r}
m \equiv<\bar{m}(E)>=\int_{0}^{\infty} \bar{m}\left(E^{\prime}\right) \cdot P\left(E^{\prime} \mid x\right) d E^{\prime}= \\
=B \cdot\left(\frac{T}{\epsilon}\right)^{\alpha} \cdot \frac{\Gamma(\alpha+1) \Gamma(\gamma-\alpha)}{\Gamma(\gamma)} \cdot \frac{{ }_{2} F_{1}(1, \alpha+1 ; \gamma+1 ; Z)}{{ }_{2} F_{1}(1,1 ; \gamma+1 ; Z)}
\end{array}
$$

or, writing this relation in different way

$$
\begin{aligned}
& \Phi(\gamma, m \mid \alpha, B, T, K) \equiv \\
& \equiv \frac{B}{m} \cdot\left(\frac{T}{\epsilon}\right)^{\alpha} \cdot \frac{\Gamma(\alpha+1) \Gamma(\gamma-\alpha)}{\Gamma(\gamma)} \cdot \frac{{ }_{2} F_{1}(1, \alpha+1 ; \gamma+1 ; Z)}{{ }_{2} F_{1}(1,1 ; \gamma+1 ; Z)}-1=0 .
\end{aligned}
$$

Equation (4.3) defines the implicit function $\gamma(m \mid \alpha, B, T, K)$. This equation is to be solved numerically preliminarily being brought to the form convenient for the iteration method:

$$
\gamma=1+G \cdot \frac{\Gamma(\gamma-\alpha)}{\Gamma(\gamma-1)} \cdot \frac{{ }_{2} F_{1}(1, \alpha+1 ; \gamma+1 ; Z)}{{ }_{2} F_{1}(1,1 ; \gamma+1 ; Z)}, \quad \gamma>1
$$

Here the value

$$
G \equiv \frac{B}{m} \cdot\left(\frac{T}{\epsilon}\right)^{\alpha} \cdot \Gamma(\alpha+1)
$$

contains the full experimental information on the sample under investigation. The properties of the detector are reflected both in value of $G$ and in the parameters $\alpha$ and $Z$ (see (3.7)). The iteration process (4.4) converges rapidly. It is convenient to start with the value $\gamma=2.0$. 
The exponent $\gamma$ evaluated from (4.4) being inserted in (3.6) defines the searched quantity of the single muon mean energy in the sample under investigation.

So the task is naturally divided into two stages. The first one is calibration of the device, i.e. the procedure of evaluation of the basic parameters $(\alpha, B, T$ and $K)$. The second step is application of calibrated device actually to the main task - estimation of separate muon mean energy in the sample of penetrating events.

\section{Calibration of device.}

As one can easily see from above, the goal of our calculations - a pair of quantities $\gamma$ and $E \equiv<E>$ - is completely fixed by the measurable quantity $m \equiv<\bar{m}>$ and by the set of device parameters represented below by the algebraic vector $f \equiv(\alpha, B, T, K)$. Further the description of the method will be accompanied by numerical illustration applied to our installation (see "Introduction").

Parameter $\alpha$ in (4.1) is obtained through calculations and does not depend on other parameters. Parameters $T$ and $K$ are obtained from (3.5). They do correlate strongly and are defined by properties of the filter above the installation and the values of $T_{0}$ and $K_{0}$ quantities for atmospheric muons.

The parameter of proportionality $B$ is estimated from the precise value of measured mean burst $m_{1}$ in events with single cosmic ray muons. The spectrum (3.1) of single muons in atmosphere is well known [6] and has the exponent value $\hat{\gamma}_{1}=2.65 \pm 0.05$. The calibration measurements of mean accompanying burst valuation $\hat{m}_{1}$ for the threshold energy $\hat{T}=35 \pm 2 \mathrm{GeV}$ of registered single muons have been carried out (the filter thickness is known with accuracy of $\simeq 5 \%$ ). For the sample of 150000 single cosmic ray muons the value of mean burst for the considered detector appeared to be $\hat{m}_{1}=$ $0.173 \pm 0.002$ (the error is statistical only).

From (4.2) it follows:

$$
\begin{array}{r}
B \equiv B\left(m_{1}, \gamma_{1}, \alpha, T, K\right)= \\
=m_{1}\left(\frac{T}{\epsilon}\right)^{-\alpha} \cdot \frac{\Gamma\left(\gamma_{1}\right)}{\Gamma(\alpha+1) \Gamma\left(\gamma_{1}-\alpha\right)} \cdot \frac{{ }_{2} F_{1}\left(1,1, \gamma_{1}+1, Z\right)}{{ }_{2} F_{1}\left(1, \alpha+1, \gamma_{1}+1, Z\right)} .
\end{array}
$$

Thus the vector of installation parameters $f(\alpha, B, T, K)$ is to be calculated through the vector of initial parameters 


$$
p \equiv\left(b, C, x, T_{0}, K_{0}, \alpha, \gamma_{1}, m_{1}\right),
$$

which were defined above.

Since all the eight parameters of $p$ vector are to be defined from independent experiments it seems to be reasonable to accept the approach to these valuations as to normally distributed independent random quantities. For the particular case of our installation we accept:

$$
\hat{p}=\left\{\begin{array}{ccccc}
p_{1}: & \hat{b}= & (4.0 \pm 0.4) \cdot 10^{-6} & \mathrm{~cm}^{2} \cdot \mathrm{g}^{-1} & {[10]} \\
p_{2}: & \hat{C}= & 460.0 \pm 46.2 & \mathrm{GeV} & {[[0]} \\
p_{3}: & \hat{x}= & 19000.0 \pm 950.0 & \mathrm{~g} \cdot \mathrm{cm}^{-2} & {[[3]} \\
p_{4}: & \hat{T}_{0}= & 1.50 \pm 0.15 & \mathrm{GeV} & {[6]} \\
p_{5}: & \hat{K}_{0}= & 110.0 \pm 5.50 & \mathrm{GeV} & {[6]} \\
p_{6}: & \hat{\alpha}= & 0.90 \pm 0.05 & & \\
p_{7}: & \hat{\gamma}_{1}= & 2.65 \pm 0.05 & & {[6]} \\
p_{8}: & \hat{m}_{1}= & 0.173 \pm 0.002 & &
\end{array}\right.
$$

The transition from initial parameters $p$ to consolidated parameters $f(p)$ for the normally distributed quantities $\hat{p}$ with covariation matrix valuation $\hat{V}$ (in this case diagonal one) is reduced to calculation of valuation

$$
\hat{f}_{\mu}=f_{\mu}(\hat{p})+\left.\frac{1}{2} \sum_{i, j=1}^{8} \hat{V}_{i j} \cdot \frac{\partial^{2} f_{\mu}}{\partial p_{i} \partial p_{j}}\right|_{\hat{p}}, \quad \mu=1,2,3,4 .
$$

Here a correction proportional to the second derivatives (5.4) of $f(p)$ is small, so all such corrections are further neglected. The covariation matrix of the valuations of consolidated parameters

$$
\hat{D}_{\mu \nu}=\left.\left.\sum_{i, j=1}^{8} \hat{V}_{i j} \cdot \frac{\partial f_{\mu}}{\partial p_{i}}\right|_{\hat{p}} \cdot \frac{\partial f_{\nu}}{\partial p_{j}}\right|_{\hat{p}}
$$

naturally is nondiagonal one. Here at least some of the derivatives above are to be calculated numerically. The quantities of valuations of consolidated parameters and of matrix $D$ are given in Table 1 .

Besides, in Fig. 1 the dependence $B(\alpha)$ is given for the device under consideration. Here the error for $\alpha$ is taken to be zero. The dashed lines are the borders of one standard deviation.

According to (3.6) one obtains for cosmic ray single muons at the threshold energy $\hat{T}=35 \mathrm{GeV}$ the value $\hat{E}_{1}=(29.8 \pm 1.9) \mathrm{GeV}$. Note that valuation of $\hat{E}$ at $\hat{T}=35 \mathrm{GeV}$ directly from the experimental data of highly precise work [ए]] leads to the value $\hat{E}_{1}^{\prime}=(32.9 \pm 2.0) \mathrm{GeV}$. 


\section{Evaluation of the exponent $\gamma$ and of the mean en- ergy $E$ of muons from the sample.}

Let us accept that for a sample of penetrating events the experimentally determined mean burst $\hat{m}$ is a normally distributed random quantity. This is approximately valid for sufficiently large samples. In such case when one obtains the valuations of $\hat{\gamma}$ and $\hat{E}$ through (4.3) and (3.6). It is possible to consider them as a substitution of random variables analogous to (5.4) and (5.5). Neglecting the corrections to mean values (as we did above) at calculation of $\hat{\gamma}$ we restrict ourself to the solution of equation (1.3) (the error of calculation must be smaller than statistical one for $\hat{\gamma}$ - see below). Namely this value of $\hat{\gamma}$ we will use to obtain the valuation of $\hat{E}$ through the relation (3.6). Since the vector of consolidated parameters $\hat{f}$ and valuation of mean burst $\hat{m}$ are statistically mutually independent, it is easy to obtain the following formulae for the dispersion of $\hat{\gamma}$ and for covariation of $\hat{\gamma}$ with $\hat{f}$ :

$$
\begin{array}{r}
\hat{\Delta}(\gamma)=\left.\left.\sum_{\mu, \nu=1}^{4} \hat{D}_{\mu \nu} \cdot \frac{\partial \gamma}{\partial f_{\mu}}\right|_{\hat{f}, \hat{m}} \cdot \frac{\partial \gamma}{\partial f_{\nu}}\right|_{\hat{f}, \hat{m}}+\hat{\sigma}_{m}^{2} \cdot\left[\frac{\partial \gamma}{\partial m}\right]_{\hat{f}, \hat{m}}^{2} ; \\
\hat{\Delta}\left(\gamma, f_{\rho}\right)=\left.\left.\sum_{\mu, \nu=1}^{4} \hat{D}_{\mu \nu} \cdot \frac{\partial \gamma}{\partial f_{\mu}}\right|_{\hat{f}, \hat{m}} \cdot \frac{\partial f_{\rho}}{\partial f_{\nu}}\right|_{\hat{f}, \hat{m}} ; \\
\rho=1,2,3,4 .
\end{array}
$$

Here $\hat{\sigma}_{m}^{2}$ is the valuation of dispersion of the mean burst $\hat{m}$ which is got from the experiment. Derivatives of $\gamma(f, n)$ are calculated from (4.3) as a derivatives of implicit function:

$$
\begin{array}{r}
\frac{\partial \gamma}{\partial f_{\nu}}=-\frac{\partial \Phi / \partial f_{\nu}}{\partial \Phi / \partial \gamma} \\
\frac{\partial \gamma}{\partial m}=-\frac{\partial \Phi / \partial m}{\partial \Phi / \partial \gamma} \\
\nu=1,2,3,4
\end{array}
$$

The dispersion of mean energy valuation for muons of the sample is obtained analogously $(E=E(f, \gamma)$, see (3.6) $)$ :

$$
\hat{\sigma}_{E}^{2}=\left.\left.\sum_{\mu, \nu=1}^{4} \hat{D}_{\mu \nu} \cdot \frac{\partial E}{\partial f_{\mu}}\right|_{\hat{f}, \hat{\gamma}} \cdot \frac{\partial E}{\partial f_{\nu}}\right|_{\hat{f}, \hat{\gamma}}+
$$




$$
\begin{array}{r}
+\left.\left.2 \cdot \sum_{\rho=1}^{4} \hat{\Delta}\left(\gamma, f_{\rho}\right) \cdot \frac{\partial E}{\partial f_{\rho}}\right|_{\hat{f}, \hat{\gamma}} \cdot \frac{\partial E}{\partial \gamma}\right|_{\hat{f}, \hat{\gamma}}+ \\
+\hat{\Delta}(\gamma)\left[\frac{\partial E}{\partial \gamma}\right]^{2}
\end{array}
$$

For example the sample of 4 -muon events with the mean burst $\hat{m}_{4}=0.45 \pm$ 0.07 leads to the valuations of $\gamma_{4}$ and $E_{4}$ :

$$
\hat{\gamma}_{4}=1.65 \pm 0.13 \quad, \quad \hat{E}_{4}=91 \pm 21 G e V .
$$

In Fig. 2 the set of dependencies $E(m)$ is given for the set of values $\alpha=$ $0.80 ; 0.85 ; 0.90 ; 1.00$ - this parameter may vary most probably in different devices (if one pais no attention to the possibility of soil-thickness variation above the installation). Here $\sigma_{m}=0, \sigma_{\alpha}=0$ is accepted. It is easy to see that in the region of relatively small energies (bursts) the result is not very sensitive to the variations of $\alpha$.

In Fig. 3 the dependencies $\gamma(m)$ and $E(m)$ are given for the above quoted values of consolidated parameters $f$ and for $\sigma_{m}=0$, i.e. the corridor of standard deviations indicated for central values of mean bursts is the systematic error of the detector. Separately standing points on Fig. 3 are the following:

1. - direct evaluation of exponent $\hat{\beta}_{4}$ in sample of 4-muon events for the case of exponential cutoff of the spectrum in the area of large bursts.

2. - value $\hat{E}_{4}$, calculated according to (3.6) for the above-mentioned value of $\hat{\beta}_{4}$ by identification $\hat{\gamma}_{4}=\hat{\beta}_{4}$ (see "Introduction").

3. - direct evaluation of exponent $\hat{\beta}_{4}^{\prime}$ in sample of 4 -muon events for purely power spectrum of bursts (argument $m$ is calculated from this spectrum).

4. - value $\hat{E}_{4}^{\prime}$ corresponding to the equality $\hat{\gamma}_{4}^{\prime}=\hat{\beta}_{4}^{\prime}$ is calculated according to $(2.3)$.

Only statistical errors are given for these four points, as well as for the central point, got by the regular method proposed.

Note that evaluation of $E$ is rather stable in respect to variation of spectrum shape at large bursts. In particular if one takes for spectrum in the sample the power approximation (2.1) but estimates $m$ neglecting the very large bursts, the values of $E$ obtained for close $m$-s differ insignificantly. 


\section{Conclusion.}

The proposed method for muon mean energy estimation with the help of accompanying burst mean value $m$ of the sample of penetrating events is based on the solution of implicit equation (4.3) for the spectrum exponent $\gamma$. The shape of this equation certainly depends on the class of functions chosen to approximate the spectrum of sample under investigation.

It is obvious that the obtained evaluations of energy depend also on the precise definition of the class of events appertained to the sample. E.g. for the sample of penetrating events of fixed number of muons these evaluations, in general, depend on the size of installation (or its sensitive area) in which the registration of fixed number of muons is demanded to attribute the event to the indicated sample.

With account of these restrictions the described method allows the reliable estimation of muon mean energies by means of accompanying burst mean value which can be measured in experiment.

Note that for the nontrack devices the concept of the "mean burst" can be substituted by e.g. "mean number of changed-state cells", or something like it, according to applied detecting elements. Of course this will require the corresponding correction but in principle differs by nothing from the above said.

\section{Acknowledgments.}

Authors express their cincere gratitude to the technical personnel of laboratory for dedicated labor which in hard circunstances made it possible to obtain the experimental data for the calibration of the method. 


\section{References}

[1] E.V.Gedalin, N.M.Gerasimova. Zh.Exp.Teor.Fiz. 45 (1963) 565.

[2] M.F.Bibilashvili, T.T.Barnaveli, G.A.Grubelashvili, N.A.Muradova. Phys.Lett. 2 (1965) 175.

[3] T.T.Barnaveli, I.V.Khaldeeva, Z.T.Shergelashvili, N.A.Eristavi. Phys.Lett. 346B (1995) 178.

[4] T.T.Barnaveli, I.V.Khaldeeva, N.A.Eristavi., in: Voprosy Atomnoi Nauki i Tekhniki. Yerevan. 5(31) (1986) 66.

[5] E.V.Gedalin. in: "Nuclear Interactions at High Energies". ed. (Metsniereba, Tbilisi, 1966).

[6] V.S.Murzin. "Introduction to the Cosmic Ray Physics". (Moscow Univ. Press. Moscow, 1988).

[7] "Investigation of Superhigh Energy Muons". ed. G.T.Zatsepin (Nauka, Moscow, 1975).

[8] S.Z.Belenky. "Avelanche Processes in Cosmic Rays". (OGIZ, Moscow, 1948).

[9] E.V.Gedalin. Zh.Exp.Teor.Fiz. 43 (1962) 1698.

[10] S.Hayakawa. "Cosmic Ray Physics. Nuclear and Astrophysical Aspects". (New York, 1969).

[11] O.C.Allkofer, K.Karstensen, W.D.Dau. in: Proc. of 12 ICRC, Vol.4, (1971) 1314. 
Figure Captions.

Fig.1. The calibration parameter $B$ dependence on the exponent $\alpha$ value for the installation considered.

Fig.2. The average energy $E$ sensibility to the exponent $\alpha$ variations. Only systematic erreos are shown.

Fig.3. The exponent $\gamma$ and average muon energy $E$ dependence on the mean burst $m$ of the sample. 
Consolidated Characteristics of the Device

\begin{tabular}{|c|c|c|c|c|}
\hline$\mu \nu$ & [1] & {$[2]$} & ${ }^{[3]} \mathrm{T}, \mathrm{GeV}$ & ${ }^{[4]} \mathrm{K}, \mathrm{GeV}$ \\
\hline & \multicolumn{4}{|c|}{ Consolidated Parameters $f_{\nu}$ of the Device } \\
\hline & $0.90 \pm 0.05$ & $0.0088 \pm 0.0016$ & $35.1 \pm 1.7$ & $135.6 \pm 5.3$ \\
\hline & \multicolumn{4}{|c|}{ Covariation Matrix $D_{\mu \nu}$} \\
\hline $\begin{array}{l}{[1]} \\
{[2]} \\
{[3]} \\
{[4]}\end{array}$ & $\begin{array}{c}+2.025 \cdot 10^{-3} \\
-7.153 \cdot 10^{-5} \\
0 \\
0\end{array}$ & $\begin{array}{l}-7.153 \cdot 10^{-5} \\
+2.640 \cdot 10^{-6} \\
-4.863 \cdot 10^{-4} \\
-3.572 \cdot 10^{-4}\end{array}$ & $\begin{array}{c}0 \\
-4.863 \cdot 10^{-4} \\
+2.759 \\
+2.227\end{array}$ & $\begin{array}{c}0 \\
-3.572 \cdot 10^{-4} \\
+2.227 \\
28.43\end{array}$ \\
\hline
\end{tabular}

Table 1: 